Théologiques

Théologiques

\title{
Sociologie politique et éthique urbaine
}

\section{Benoît Bégin}

Volume 3, numéro 1, mars 1995

Symbolique urbaine et foi chrétienne

URI : https://id.erudit.org/iderudit/602416ar

DOI : https://doi.org/10.7202/602416ar

Aller au sommaire du numéro

Éditeur(s)

Faculté de théologie de l'Université de Montréal

ISSN

1188-7109 (imprimé)

1492-1413 (numérique)

Découvrir la revue

Citer cet article

Bégin, B. (1995). Sociologie politique et éthique urbaine. Théologiques, 3(1), 89-101. https://doi.org/10.7202/602416ar

\section{Résumé de l'article}

Étroitement reliée à la révolution industrielle, l'urbanisation connaît aujourd'hui une expansion à une échelle absolument inconnue dans toute l'histoire de l'humanité et constitue un milieu de vie qui comporte des transformations majeures dans la manière de penser, de sentir et de vivre des personnes. Des études récentes plus radicales en sociologie et en théologie nous montrent que ce n'est pas d'abord la ville elle-même qui est la cause de l'aliénation et de l'isolement dont sont victimes les personnes et les communautés, mais avant tout le système fondamental de valeurs qui place la rationalité technologique et la concurrence économique au-dessus des valeurs culturelles et sociales. C'est le système capitaliste lui-même, à la source des maux de la ville, qui est remis en question. 
Théologiques 3/1 (1995) 89-101.

\title{
Sociologie politique et éthique urbaine
}

\author{
Benoît BÉGIN \\ Institut de Pastorale \\ Université Saint-Paul
}

\section{RÉSUMÉ}

Etroitement reliée à la révolution industrielle, l'urbanisation connaît aujourd'hui une expansion à une échelle absolument inconnue dans toute l'histoire de l'humanité et constitue un milieu de vie qui comporte des transformations majeures dans la manière de penser, de sentir et de vivre des personnes. Des études récentes plus radicales en sociologie et en théologie nous montrent que ce n'est pas d'abord la ville elle-même qui est la cause de l'aliénation et de l'isolement dont sont victimes les personnes et les communautés, mais avant tout le système fondamental de valeurs qui place la rationalité technologique et la concurrence économique au-dessus des valeurs culturelles et sociales. C'est le système capitaliste lui-même, à la source des maux de la ville, qui est remis en question.

Construire aujourd'hui la ville, lieu d'existence des hommes et de leurs communautés élargies, créer de nouveaux modes de proximité et de relations, percevoir une application originale de la justice sociale, prendre en charge cet avenir collectif qui s'annonce difficile, c'est une tâche à laquelle des chrétiens doivent participer.

( Paul VI, Octogesima Adveniens, § 12)

Avec l'industrialisation, l'urbanisation est au cœur des théories sociologiques contemporaines. La révolution industrielle est définitivement au centre de toute théorie qui veut rendre compte des changements auxquels nous sommes confrontés dans notre monde contemporain. C'est elle qui a transformé dans son sillage le phénomène urbain qui connaît aujourd'hui une expansion à une échelle absolument inconnue dans toute l'histoire de l'humanité (Giddens 1987: 5). L'urbanisation est un développement 
historique majeur dont l'ampleur est devenue universelle, au même titre que l'expansion industrielle et l'affaissement de la civilisation agraire. Les citoyens des sociétés modernes avancées ${ }^{1}$ sont désormais habitués à vivre en milieu urbain, creuset de transformation sociale dont on ne soulignera jamais assez l'importance (Giddens 1987:92). La vie urbaine, il est vrai, ne date pas des temps modernes puisqu'elle est née bien avant la révolution industrielle. Mais son explosion actuelle est un phénomène sacial nouveau ( Paul VI 1971 : 79; Giddens 1987: 5 ).

Ce phénomène contemporain majeur comporte des éléments économiques, sociaux et politiques qui ont un impact sur la vie des hommes et des femmes de cette période historique particulière que nous vivons. Étroitement reliée au développement industriel, l'urbanisation, comme lui, est à la source de comportements nouveaux dans les relations humaines (Gaudium et Spes : $\$ 54$ ). Les efforts des hommes et des femmes vers l'orientation de leur avenir sont en effet liés aux conditions de vie courantes. C'est pourquoi l'existence humaine ne peut se comprendre sans faire appel aux institutions qui la rendent possible (Mills 1971: 161170).

Il importe donc, si nous voulons entreprendre une action concrète en vue du bien des personnes et des communautés, d'aborder les nouvelles conditions de vie dans leur cadre le plus global, c'est-à-dire dans un contexte d'économie moderne et de civilisation nouvelle. C'est là qu'on trouve la clé des problèmes sociaux nouveaux reliés à la ville. Les changements sociaux et structurels posent à la théologie et à l'éthique des questions fondamentales qui nous incitent à lire et discerner les "signes des temps ", à nous ouvrir aux éléments dialectiques qui entrent en jeu dans la facture même de notre société, et à mettre en œuvre un dialogue serré avec la réalité du monde urbain moderne. Désormais l'activité humaine dans l'univers, qu'elle soit civile ou pastorale, doit s'analyser en fonction des grandes structures socio-historiques qui constituent le contexte au cœur duquel s'enracine la vie des hommes et des femmes d'aujourd'hui. La société contemporaine constitue un milieu de vie qui transforme les per-

1 Le sociologue Anthony Giddens emploie les termes high modernity ou late modernity, traduits en français par "modernité avancée ". Selon Giddens, même si nous vivons définitivement dans une société post-traditionnelle, nous n'en sommes pas encore arrivés à la postmodernité, comme trop d'auteurs semblent le croire. Le terme postmodernité peut facilement justifier des points de vue idéologiques. Voir GIDDENS $1992: 3-4 ; 1991$. 
sonnes, leur manière de penser et de sentir et plus profondément encore leur système de valeurs.

\section{Contexte historique de la ville}

Prenons acte du fait qu'on ne peut séparer l'étude du phénomène urbain de celle de l'évolution de notre société industrielle dans une modernité avancée. C'est là qu'il faut chercher les coordonnées de vie selon lesquelles nous fonctionnons aujourd'hui.

Or, nous vivons à une époque de transformation sociale massive. En l'espace d'à peine deux siècles, une avalanche de changements sociaux se sont produits et continuent à se succéder avec toujours plus de force, au point de dissoudre presque complètement les modèles d'organisation sociale selon lesquels l'humanité s'était habituée à vivre durant des milliers d'années de son histoire (Giddens 1987:5).

Avant le XIX ${ }^{e}$ siècle, même les sociétés les plus urbanisées ne comptaient pas plus que $10 \%$ de leur population vivant dans des villes ou cités. Par rapport à nos standards modernes d'évaluation, à peu près toutes les villes des sociétés pré-industrielles, même les centres cosmopolites les plus connus, étaient relativement peu peuplés. Londres, par exemple, ne comptait pas plus que 30000 âmes au Moyen Âge, et Florence, au temps de la Renaissance, 90000 . Aujourd'hui, au contraire, tous les pays industrialisés sont largement urbanisés (Giddens 1987: 5-6). Et c'est dans la forme et l'étendue de la ville moderne que se manifestent le plus les changements profonds qui ont transformé la vie sociale mondiale depuis 200 ans. Ces mutations urbaines sont devenues le milieu de vie de la majorité des hommes et femmes des sociétés capitalistes avancées (Giddens 1987 : 92 ). Soulignons surtout que ces développements ne furent pas uniquement quantitatifs, comme on semble le prétendre dans certaines Écoles de sociologie nord-américaines. L'urbanisation reliée au capitalisme industriel fut beaucoup plus qu'un mouvement de migration des régions rurales vers les villes. Elle comporte toute une panoplie d'autres changements qui touchent la nature même du mode urbain de vie ( Giddens 1987 : 94 ).

\section{Théorie urbaine classique}

Il serait trop facile de revenir à la dichotomie ville/campagne, qui a marqué les principaux courants de sociologie urbaine, pour expliquer tous les problèmes générés par la ville moderne. Nous devons pourtant relever les principales données de cette théorie classique. 
Son thème central, reliant les principales écoles de ce courant de pensée, c'est le passage d'une société traditionnelle, morale et personnelle, à une société moderne, instrumentale et impersonnelle. La thèse proposée, exprimée de façon très succincte, est la perte du sens communautaire comme résultat des changements profonds causés par le développement des grands centres urbains industriels.

La thèse manifeste une nostalgie du passé et la vision d'une forme plus humaine de communauté comme foyer de relations harmonieuses, intégrées et stables, ainsi que de sentiments collectifs de loyauté et d'appartenance. En contraste, le monde présent se définit en termes d'individualisme grandissant marqué d'un manque d'harmonie, de désintégration, d'instabilité, de déloyauté et de manque de sentiment d'appartenance. Foncièrement, la thèse constitue une critique fondamentale de l'industrialisation et de la vie urbaine (Hale 1990: 107 ).

\subsection{Tönnies : Gemeinschaft et Gesellschaft}

Cette théorie classique remonte au sociologue et philosophe allemand Ferdinand Tönnies qui, le premier, dans son étude Gemeinschaft und Gesellschaft ( 1887 ), exposa la thèse de la perte de la communauté. À la suggestion de la sociologue Sylvia Hale, on peut traduire de façon assez libre le terme Gemeinschaft par "communauté " et Gesellschaft par "association ". Ainsi, avec le développement de la société moderne industrielle émergea un modèle du tout au tout différent de celui de la société préexistante qu'il remplaçait. Ce fut la Gesellschaft, une association d'individus motivés par un intérêt calculé s'inscrivant dans un esprit de capitalisme compétitif, de poursuite rationnelle de profit et d'avantage individuel. Ainsi, arguait le sociologue allemand, l'industrialisation qui marqua fondamentalement la société engendra des relations déshumanisées et artificielles ( Hale 1990: 99 ).

\subsection{L'École de Chicago}

L'École de sociologie urbaine de Chicago, qui vit le jour dans les années 1920, s'inspira des conceptions de Tönnies dans l'élaboration d'une théorie générale de la vie urbaine. Acceptant la classification de base des types rural et urbain, ses théoriciens présentèrent des positions de rechange. Ainsi, par exemple, Louis Wirth, dans son essai "Urbanism as a Way of Life " ( 1938 ), mit sur pied une théorie suggérant que la cité en tant que cité recelait des caractéristiques expliquant les modèles de culture identifiés par Tönnies ( Hale 1990: 109; Giddens 1987: 99) entre autres. Plus clairement, les deux sociétés successives mises en contraste 
par celui-ci furent alors identifiées comme étant le rural et l'urbain. Nous verrons que l'équation Gesellschaft/vie urbaine est difficilement soutenable : de nouvelles approches en sociologie politique (political economy perspective) se sont chargées de remettre en question cette théorie.

Pour Wirth, trois variables causent différentes déterminations dans la communauté : son volume, sa densité et son hétérogénéité ( Hale 1990 : 109-111; Giddens 1987: 96-97).

La dimension excessive d'un centre urbain engendre une différentiation accrue des individus qui le composent. Il leur devient impossible de se connaître et d'échanger entre eux sur une grande variété d'intérêts. Les interactions communautaires en deviennent plus limitées et pour autant superficielles, transitoires et anonymes. D'où la solitude de l'individu au milieu de la foule urbaine.

Une trop grande densité de nos villes produit un effet également néfaste. Le surpeuplement entraîne, outre la pollution de l'air, celle des rapports interpersonnels. Il en va de la cité trop populeuse comme de ces laboratoires où des rats, artificiellement entassés les uns sur les autres, se battent pour défendre leur territoire, alors que dans une cage spacieuse ils vivraient en paix. L'agressivité qu'ils démontrent dans un espace vital restreint ressemble à l'attitude anti-sociale des individus humains entassés dans une ville.

Enfin le caractère hétérogène de nos cités tend à miner le sentiment d'appartenance des individus à leur communauté ou à leur groupe. La promiscuité de gens ethniquement différents, détenant un statut différent, des occupations différentes, des mentalités différentes ne peut produire que des allégeances différentes, des incompréhensions, des intolérances.

Il faut remarquer que cette conception de l'École de Chicago renverse celle de Tönnies dont elle s'inspire pourtant. Pour ce dernier, en effet, c'est la poursuite rationnelle et égoïste d'avantages économiques qui cause l'effondrement de la communauté et provoque subséquemment l'exode rural vers la cité. Pour l'École de Chicago, au contraire, c'est l'entassement dans les cités qui produit l'effritement de la communauté et la situation d'anomie particuliers à la Gesellschaft.

Sylvia Hale remarque pertinemment que somme toute, la position de Tönnies et ses amendements par l'École de Chicago relèvent d'une idéologie essentiellement conservatrice: la vie de village avec l'accent qu'elle met sur la famille, l'église et la tradition apparaît plus naturelle et stable, tandis que la vie urbaine semble plus artificielle et instable. L'impression qui résulte de l'étude de ces théories en est une de perte et 
de régression: le concept de communauté effritée suscite un sentiment d'insatisfaction: la qualité de vie du monde contemporain apparaît décevante, et le désir se fait plus pressant de retourner à une société plus humaine formée d'individus vivant dans une communauté stable de famille, d'amis et de voisins. Le concept de communauté dit sécurité et assurance ainsi qu'identité et appartenance. La sociologue ajoute que même si le passé peut bien n'avoir pas été aussi rose que l'image idéalisée de la vie communautaire le représente, le sentiment de perte reste un phénomène social à ne pas mésestimer dans la mesure où il contient une critique implicite du présent et démontre les difficultés ressenties par les gens. À ce titre, il doit faire l'objet d'une analyse sociologique sérieuse ( Hale $1990: 110-111$ ).

\section{Théorie critique}

Pour mieux saisir le sens des changements radicaux qui se sont opérés dans nos cités modernes, nous devons situer le phénomène de l'urbanisation dans le cadre plus global du développement industriel capitaliste. Le type de cité qui a précédé l'avènement du capitalisme était surtout un chef-lieu d'opérations gouvernementales, son activité productive et commerciale étant plutôt limitée. La majorité de la population était alors engagée en agriculture. C'est l'émergence du capitalisme et sa consolidation industrielle surtout qui occasionnèrent l'exode massif des ruraux vers les milieux urbains, et en modifièrent la structure profonde (Giddens $1987: 100)$.

Une théorie critique de conception marxienne établit clairement la relation qui existe entre les transformations radicales que subit la cité contemporaine et le développement du capitalisme : l'ordre capitaliste signifie l'achat et la vente de marchandises, y compris la force de travail, dans le but de réaliser des profits. Le libéralisme économique applique au maximum ce principe de base aux réalités qui constituent le milieu humain. C'est ainsi que l'espace lui-même devient une marchandise susceptible de commerce : la terre et l'habitation qui préalablement étaient inaliénables, c'est-à-dire non susceptibles de transfert d'une personne à l'autre moyennant paiement, font désormais partie des choses susceptibles d'offre et de demande (Giddens 1987 : 101 ).

Cette commercialisation de l'espace entraîne une complexification considérable du milieu physique. Première conséquence : l'industrie s'étend partout, y compris dans les campagnes qui, de plus en plus, ne se démarquent pas essentiellement des villes. L'agriculture elle-même fonctionne désormais comme une entreprise capitaliste, et, pour une efficacité 
rentable, se mécanise à l'instar des autres secteurs industriels. Et ce changement dans le mode de production crée des changements dans les modes de vie sociale; de ce point de vue également, la différence entre le rural et l'urbain s'abolit. Mais allons plus loin encore: les personnes qui travaillent dans un milieu créé par le capitalisme industriel et qui vivent dans un espace devenu objet de commerce, se séparent d'autant de la nature dans leur façon de vivre. C'est que l'atmosphère qui entoure l'humain est devenue un environnement au service de l'usine et du bureau, coupé à toute fin pratique de la nature vivante que représentent la végétation, la météo et le cycle des saisons (Giddens 1987: 102).

La progression du capitalisme industriel au cœur de la société moderne, tout particulièrement dans l'organisation même de la vie urbaine, a pour conséquence de dépouiller la vie quotidienne de ce que Lefebvre appelle "la poésie de la vie " et de lui donner, selon Giddens, un caractère routinier et prosaïque, coupé de valeurs morales et vivantes : ce qui prime avant tout, c'est l'économique, l'instrumental et le technique (Giddens 1987 : 112-113). La position marxienne rejoint sans doute ici celle de Tönnies et de l'École de Chicago quand il s'agit de constater l'effet déshumanisant de la société capitaliste, et la perte de sentiment communautaire et d'appartenance qu'elle entraîne, mais elle explique le phénomène à partir d'autres facteurs.

Une autre différence majeure avec l'École de Chicago qu'apporte la théorie critique marxienne a trait à la sécurité économique : les personnes qui contrôlent leurs moyens de production peuvent entretenir des relations sociales satisfaisantes. Ici "la perte de la communauté ", indépendamment de la ville ou de la campagne, s'explique par un environnement économique déficient. Dès lors, indépendamment de leur volume ou endroit, les communautés qui montreront les signes les plus évidents de désorganisation sociale et de démoralisation seront celles qui seront frappées par une pauvreté relativement sévère, et dont les habitants auront perdu leur sens de sécurité économique et de contrôle de leur situation.

La qualité de vie des personnes et le bien-être des communautés ne s'évaluent pas nécessairement par la démographie. À partir des trois facteurs de volume, de densité et d'hétérogénéité avancés par Wirth, les exemples ne manquent pas, pour le contredire, de villages restreints à ce triple point de vue, peuplés de gens qui connaissent la solitude, l'isolation et la méfiance. Les exemples ne manquent pas non plus de personnes qui, vivant dans un milieu métropolitain très étendu et populeux, disent goûter une existence communautaire intégrée avec des liens très forts de voisinage et de parenté ( Hale $1990: 127-128$ ). 
La théorie d'économie politique souligne à bon droit l'influence de la structure industrielle capitaliste, et invite à explorer la force débilitante d'un système économique qui crée l'appauvrissement, le chômage, la condition semi-prolétarienne, la dépendance morbide. Ce dont les gens ont besoin pour garder l'équilibre de leur vie de communauté, c'est de cette confiance de base que crée une situation de sécurité économique. Pourtant, il ne faut pas durcir à l'extrême cette conception, au risque de tomber dans un déterminisme économique prédisant la désorganisation sociale de toute communauté où sévirait la pauvreté. Malgré la puissance de ce facteur, des personnes et des groupes ont maintes fois prouvé leur capacité de résister aux courants d'aliénation sociale et de maintenir malgré tout la vitalité de leur communauté ( Hale 1987: 132). Ce que l'on remarque en fin de compte, c'est que la théorie critique marxienne est beaucoup plus proche des théories explicatives originales de Tönnies sur la différence entre Gemeinschaft et Gesellschaft que ne l'était l'École de Chicago. En déplorant la perte de la communauté, Tönnies ne s'attaquait pas d'abord à la vie urbaine comme telle, à la taille et à la composition de la communauté. Il s'en prenait avant tout à un système qui place la rationalité technologique et la concurrence économique au-dessus des valeurs culturelles et sociales. Dans ce sens, la théorie critique marxienne nous éclaire par sa remise en question des valeurs du système capitaliste lui-même à la source des maux de la ville (Hale 1990: 135 ).

\section{4. Éthique urbaine}

L'analyse sociologique de la ville que nous avons présentée jusqu'ici, loin de venir en conflit avec une éthique chrétienne de la ville ou une "théologie de la ville» (Comblin 1968), nous semble au contraire fournir des fondements sociaux scientifiques pour le renforcement de l'approche actuelle en réflexion théologique. On ne peut plus, en effet, s'en tenir aux théories traditionnelles qui ont trop longtemps eu cours en sociologie comme en théologie, et au cœur desquelles on retrouve presque toujours l'affrontement ville-campagne. Surtout quand il s'agit du Québec où le phénomène de l'urbanisation s'est produit avec un retard de quelques générations. La théorie classique d'opposition entre campagne et ville sur le modèle de l'opposition entre nature et société n'est plus tenable aujourd'hui (Bookchin 1992 : xxiv). Lorsqu'il est question d'habitat humain et de communauté humaine, il n'est pas plus "naturel " de vivre à la campagne que de vivre en ville. Le monde biologique et le monde social sont tous deux "naturels" en un sens très réel. Et ce qui est " naturel " à la ville, c'est d'être une production humaine, une institution politique qui appartient au domaine de la culture. Les institutions sociales, 
en effet, comme l'affirme magistralement l'ethnologue Claude LéviStrauss, sont des synthèses d'un ordre nouveau entre la nature et la culture, qui permettent à la vie humaine de se développer dans toute sa richesse (Lévi-Strauss 1968: 3-4). La différence entre ville et campagne, dans la mesure où elles constituent des milieux humains, est en ce sens abolie.

Les études récentes, plus réalistes, gardent un regard critique sur le développement urbain contemporain, mais considèrent d'emblée que la ville représente l'avenir de l'humanité, tout comme elle a été, à un degré considérable dans les millénaires passés, le creuset des civilisations (Bookchin 1992: xxv). C'est dans les villes du Proche-Orient et de l'Europe méridionale, ne l'oublions pas, qu'est né le christianisme. S'il en est ainsi, on serait mal venu d'opposer humanisme et ville.

Ce n'est pas l'existence même de la ville qui a créé la crise urbaine, mais plutôt "l'émergence d'un phénomène relativement nouveau et cancéreux qui menace de mort tant la cité que la campagne : l'urbanisation " (Bookchin 1992 x). Au dire de Murray Bookchin, considéré aujourd'hui comme l'un des meilleurs spécialistes en écologie urbaine ${ }^{2}$, l'urbanisation est une réalité moderne issue de la dynamique propre à la cité traditionnelle. C'est à partir des changements institutionnels, technologiques et sociaux qui ont marqué nos sociétés contemporaines qu'elle s'en est séparée pour assumer son propre développement et ravager tant l'écologie de la cité que l'agriculture de la campagne. Ces transformations ont entraîné dans leur sillage une diminution du rôle décisionnel des citoyens, en réduisant à toute fin pratique leur importance à celle du payeur de taxes et de l'électeur (Bookchin 1992: 12).

La conséquence la plus grave de cet état de fait, c'est le retrait des citoyens de la vie sociale et politique et leur réduction éventuelle à la passivité et à la consommation. C'est ce qu'exprime Ricœur dans une formule bien marquée:

Que la plupart de nos contemporains se pensent d'abord comme consommateurs, puis comme travailleurs, enfin seulement comme citoyens, ce n'est là que le signe le plus voyant, le plus caricatural, de l'auto-déception d'un grand projet (Ricceur 1989:492-93).

2 Kent Gerecke, professeur à l'Université du Manitoba et rédacteur de City Magazine affirme que Bookchin fait aujourd'hui figure de proue en écologie urbaine: son oeuvre rejoindrait les travaux gigantesques de Lewis Mumford sur la culture et l'histoire des cités, les dépassant même. 
Le dépouillement du pouvoir social et politique conduit inévitablement à la déshumanisation et à la perte même de l'identité des personnes. C'est donc ainsi, en bref, que l'urbanisation a pu éventuellement balayer toutes les institutions civiques et agraires qui assuraient à l'individu une portion d'autonomie.

On peut facilement jauger la teneur éthique d'un tel phénomène : la conviction de base qui le sous-tend, tout à fait dans la ligne du libéralisme, est le progrès économique comme finalité ultime et incontestable de la société, et le profit à tout prix comme clef de voûte. De là la loi de la compétition et de la propriété privée considérées comme un droit absolu, sans limites ni obligations sociales (Sheridan 1987:330). En plus d'inhiber le sens du partage, de solidarité et d'amour de nos communautés, un tel système est facteur de contradictions sociales patentes : la production et le profit à tout prix incitent systématiquement à la surproduction et la surconsommation au détriment des ressources naturelles et écologiques. Nous en sommes rendus à un tournant où le phénomène d'urbanisation selon un modèle d'économie de marché capitaliste ne menace plus seulement la cité en tant que telle, mais l'environnement global de l'existence humaine. Cette menace ne vient pas seulement de l'empiétement géographique des développement urbains, mais de façon encore plus critique de la déshumanisation, autant des modes de vie urbaine que des modes de vie rurale: c'est en fait la destruction dans ses fondements même de la communauté humaine et vivante. Dans la ligne de l'enseignement social de l'Église, les évêques du Canada déclarent que «les réalités économiques actuelles de notre société révèlent un désordre moral... [et que] nous avons la responsabilité de soulever les problèmes sociaux et éthiques qu'elles comportent "( Sheridan 1987: 402). Et, de son côté, Bookchin rappelle jusqu'à quel point la ville doit être au service des citoyens :

[...] la ville, à son meilleur, [doit devenir] une union éthique de personnes, une écono-communauté éthique et sociale, et non pas simplement un ensemble dense de structures destinées à fournir des biens et services à des résidents anonymes (Bookchin 1992: x ).

Dès lors, dans les termes du même auteur, l'intervention en milieu urbain, qu'elle soit pastorale ou strictement sociale, aura pour tâche de

réhabiliter la ville, l'explorer non pas comme un phénomène corrosif, mais plutôt comme une communauté humaine, éthique et écologique à un degré unique, dont les membres ont longtemps vécu en équilibre avec la nature et les formes institutionnelles créées, ce qui a aiguisé la conscience de soi de l'humain, nourri sa rationalité, produit sa culture 
sécularisée, renforcé son individualité et établi ses formes de liberté (Bookchin 1992 : xiv).

Nous retrouvons ici la notion grecque de la cité (ou polis) comme étant une union éthique de citoyens. Au lieu de s'en tenir à ce que la cité est à un moment donné de son histoire, il faut chercher ce qu'elle devrait être, en mettant en œuvre non pas les impératifs d'un credo arbitraire, mais la faculté humaine de raisonner et de tirer de ses potentialités l'édification de communautés matures, conscientes, et libres. De telles communautés seraient l'intégration en une nature dite libre de ce qu'il y a de meilleur dans la nature biologique et la nature sociale, et établiraient une interaction créatrice avec l'environnement naturel. Le but serait alors l'établissement de réseaux de villes à une échelle plus humaine, d'institutions politiques directement démocratiques permettant l'apport du citoyen au contrôle des moyens de production, et d'un idéal plus large et étendu de liberté (Bookchin 1992 : xvii-xviii).

\section{Conclusion}

À travers les quelques éléments de sociologie radicale ${ }^{3}$ qui nous ont servi de grille d'analyse pour une meilleure compréhension du milieu urbain, nous avons cru rejoindre des données importantes de la théologie politique et de ses fondements sociaux (Baum 1975; 1987) : nous avons pu reconnaître en effet les mêmes hypothèses méthodologiques et une complémentarité manifeste. En Église, on accepte de plus en plus l'importance de l'analyse sociale pour établir des programmes efficaces en pastorale (Holland et Henriot 1990). Dans un document récent, le Comité de théologie de l'Assemblée des évêques du Québec affirmait que

l'économie de marché tend à envahir tous les domaines pour y imposer sa rationalité er même se substituer au social. Elle transforme les relations sociales en relations d'affaires. Les valeurs humaines et sociales risquent d'être avalées par les valeurs marchandes : "Ce qu'il y a de plus intolérable, c'est le recul des droits sociaux et démocratiques devant ce qu'il faut bien appeler la dictature du marché $[\ldots]$ » ( $1994: 14)$.

Cette approche interdisciplinaire renforce la conviction que le modèle économique qui a envahi tous les secteurs de notre société n'est pas tout à

3 Le mot " radical » dans son sens étymologique (radix) signifie « à la racine », " à la base ». 
fait la preuve du progrès social ni le présage d'un modèle éthique plus humain.

Dans les termes d'un philosophe chrétien aussi crédible que Paul Ricœur, il y a "contradiction entre la logique de l'industrialisation et la vieille rationalité relevant de l'expérience politique des peuples ": la technologie devenue souveraine exerce une action dissolvante sur l'organisation de nos sociétés. D'où la revendication majeure à l'endroit de l'économico-technique doit être celle d'une finalité humaine inséparable "de la morale vivante, de l'intention éthique " (Ricœur 1986 : 397-398). C'est là un prérequis pour l'épanouissement d'une société démocratique au sein de laquelle les citoyens peuvent exercer une activité responsable dans la cité et contribuer à "l'avènement d'un ordre social qui ne soit plus au service d'une économie vorace et insatiable, mais plutôt au service des humains dans toutes leurs dimensions individuelles et collectives» (Comité de théologie $1994: 70$ ).

\section{Bibliographie}

BAUM, Gregory. 1975. Religion and Alienation: A Theological Reading of Sociology. New York, Paulist Press, 296 p.

BAUM, Gregory. 1987. Theology and Society, New York, Paulist Press, $298 \mathrm{p}$.

BOOKCHIN, Murray. 1992. Urbanization Without Cities. The Rise and Decline of Citizenship. Montréal/New York, Black Rose Books, 316 p.

COMBLIN, Joseph. 1968. Théologie de la ville. Paris, Éditions Universitaires, $493 \mathrm{p}$.

COMITÉ DE THÉOLOGIE DE L'ASSEMBLÉE DES ÉVÊQUES DU QUÉBEC. 1994. L'engagement des communautés chrétiennes dans la société. Montréal, Fides, 70 p.

CONCILE VATICAN II. 1966. L'Église dans le monde de ce temps. Constitution pastorale *Gaudium et Spes». Montréal, Fides, 109 p.

GIDDENS, Anthony. 1987. Sociology: A Brief but Critical Introduction. New York, Harcourt Brace Jovanovich, 179 p.

GIDDENS, Anthony. 1991. "Une théorie critique de la modernité avancée ". dans Colloque Structuration du social et modernité, Cerisyla-Salle ( texte non publié ), 29 p. 
GIDDENS, Anthony. 1992. Modernity and Self-Identity: Self and Society in the Late Modem Age. Stanford, Stanford University Press, 256 p.

HALE, Sylvia M. 1990. Controversies in Sociology: A Canadian Introduction. Toronto, Clark Pitman Ltd., $530 \mathrm{p}$.

HOLlAND, Joe, HENRIOT, Peter. 1990. Comprendre la société. Adaptation française de Social Analysis Linking Faith and Justice. Bruxelles, Vie Ouvrière, 139 p.

LÉVI-STRAUSS, Claude. 1968. Les structures élémentaires de la parenté. Paris, Mouton, $591 \mathrm{p}$.

MILLS, C. Wright. 1971. L'imagination sociologique. Traduit de l'américain par Pierre Clinquart. Paris, Maspéro, 235 p.

PAUL VI. 1971. Octogesima Adveniens. Lettre apostolique à $M$. le Cardinal Maurice Roy à l'occasion du $80^{e}$ anniversaire de * Rerum Novarum . La responsabilité politique des chrétiens. Présentation et commentaires par l'équipe des Pères de C.E.R.A.S.-Action populaire. Paris, Éditions ouvrières, 148 p.

RICCEUR, Paul. 1986. "Éthique et politique ", dans Du texte à l'action. Paris, Seuil, 393-406.

RICCEUR, Paul. 1989. "Langage politique et rhétorique ", dans Phénoménologie et Politique. Mélanges offerts à Jacques Taminiaux. Bruxelles, Ousia, 479-495.

SHERIDAN, E. F., éd. 1987. Do Justice! The Social Teaching of the Canadian Catholic Bishops. Sherbrooke, Paulines; Toronto, The Jesuit Centre for Social Faith and Justice, 470 p. 